TEMA Tend. Mat. Apl. Comput., 8, No. 1 (2007), 119-128.

(C) Uma Publicação da Sociedade Brasileira de Matemática Aplicada e Computacional.

\title{
Uma Abordagem Fuzzy para um Modelo Presa-predador Acoplado ao Parasitismo
}

M.S. PEIXOTO, Universidade Federal de São Carlos, Campus Sorocaba, 18043-970 Sorocaba, SP, Brasil

L.C. BARROS, Departamento de Matemática Aplicada, IMECC, UNICAMP, 13083-859 Campinas, SP, Brasil

R.C. BASSANEZI, Centro de Matemática, Computação e Cognição, UFABC, 09210-170 Santo André, SP, Brasil.

Resumo. Nesse trabalho utilizamos sistemas baseados em regras fuzzy para elaborar um modelo do tipo presa-predador acoplado ao parasitismo para estudar a interaçao entre pulgões (presa/hospedeiro), joaninhas (predador) e um afídideo (parasitóide) na citricultura, onde pulgões são considerados agentes transmissores da Morte Súbita dos Citros. Simulações são realizadas e analisadas por gráficos de populações de presas, potencialidade dos predadores e população de parasitóides, bem como um espaço de fase obtido por simulações.

\section{Introdução}

A Morte Súbita dos Citros é uma doença que tem afetado grande parte dos pomares de citros do Estado de São Paulo [2]. Pesquisadores acreditam que tal doença seja causada por um vírus transmitido por insetos conhecidos como pulgões, que têm como inimigos naturais uma espécie de joaninha (predador) [12] e um afidídeo (parasitóide) [14].

Nesse artigo sugerimos a Teoria dos Conjuntos Fuzzy [23] para modelar a interação entre presa/hospedeiro (pulgão), seu predador (joaninha) e seu parasitóide (afidídeo), como uma nova opção ao uso de equações diferenciais que caracterizam os modelos determinísticos clássicos. Uma vez que as informações a respeito do fenômeno são qualitativas, é difícil expressar as variações como funções dos estados. Por outro lado, tais informações, dadas por especialistas, permitem-nos elaborar regras que relacionam (ainda que parcialmente), as variáveis de estado com suas próprias variações. Nosso principal interesse é elaborar um modelo presapredador acoplado ao parasitismo que represente a interação entre pulgões (presas/hospedeiros), joaninhas (predadores) e afidídeos (parasitóide) na citricultura, por meio de sistemas baseado em regras fuzzy [1]. 


\section{Conceitos e Definições}

Um subconjunto fuzzy $A$ de um conjunto universo $X$ é definido pela função de pertinência $\psi_{A}$ que associa a cada elemento $x$ de $X$ um número $\psi_{A}(x)$, entre zero e um, que indica o grau de pertinência de $x$ a $A$. Assim, $\psi_{A}: X \rightarrow[0,1]$.

É interessante notar que um subconjunto clássico $A$ de $X$ é um conjunto fuzzy particular para o qual a função de pertinência é sua função característica, $\chi_{A}: X \rightarrow$ $\{0,1\}$.

Sistema baseado em regras fuzzy. Basicamente, um sistema baseado em regras fuzzy possui quatro componentes: um processador de entrada; uma coleção de regras lingüísticas, chamada base de regras; um método de inferência fuzzy e um processador de saída. Esses componentes processam valores reais de entrada em valores reais de saída. A Figura 1 ilustra um sistema baseado em regras fuzzy.

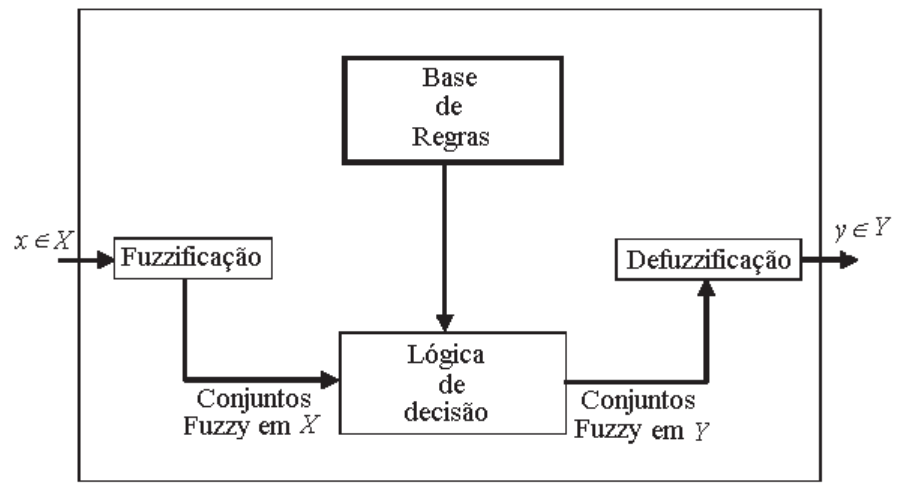

Figura 1: Estrutura de sistemas baseados em regras fuzzy [10].

A base de regras é composta por uma coleção de proposições condicionais fuzzy na forma de regras se-então. Um especialista do fenômeno analisado fornece as informações para se formular o conjunto de regras fuzzy que ativa as associações de entradas/saídas lingüísticas.

O método de inferência que utilizamos é o de Mamdani [15], que agrega as regras por meio do operador lógico OU, modelado pelo operador máximo e, em cada regra, os operadores lógicos E e ENTÃO são modelados pelo operador mínimo [11]. Um sistema baseado em regras fuzzy considerado nesse trabalho, consiste de duas variáveis de entrada, duas de saída e 25 regras (ver seção 4).

Por fim, na defuzzificação, o valor da variável lingüística de saída inferida pelas regras fuzzy é traduzida num valor real. O objetivo é obter um valor real que melhor represente os valores fuzzy de saída. O método de defuzzificação adotado nesse artigo é o do Centro de Gravidade definido como segue. Seja $C$ a função de pertinência da variável de saída $z$. Então, o valor real (defuzzificado) de saída $\bar{z}$ é 
dado como segue

$$
\bar{z}=\frac{\int z C(z) d z}{\int C(z) d z} .
$$

Sugerimos a leitura de [1],[15] e [11] para um entendimento detalhado dos fundamentos da teoria dos conjuntos e sistemas fuzzy e suas aplicações. Na próxima seção faremos uma breve revisão sobre a interação entre presas e predadores, hospedeiros e parasitóides.

\section{Predação e Parasitismo}

A predação e o parasitismo são exemplos familiares de interação entre duas populações que resultam em efeitos negativos no crescimento e sobrevivência de uma população e em um efeito positivo ou benéfico na outra. O predador mata a presa para se alimentar. Entendese por parasitóide a espécie que parasita outra somente os estágios imaturos, matando o hospedeiro durante o seu processo de desenvolvimento, vivendo livre quando adulto.Do ponto de vista ecológico, podemos dizer que predação e parasitismo são semelhantes [3].

Os modelos matemáticos que descrevem relações do tipo presa-predador ou hospedeiro-parasitóide são usados para estudar interações entre populações, onde uma depende da outra para se alimentar ou reproduzir e sobreviver. Tais relações dinâmicas entre presas e predadores e entre hospedeiros e parasitóides são objetos de estudo proeminente em Ecologia [5]; [13].

A seguir apresentamos hipóteses que caracterizam um modelo presa-predador (hospedeiro-parasitóide), cujas trajetórias têm as seguintes características:

1. A quantidade de presas e a quantidade de predadores têm um caráter oscilatório;

2. Um crescimento na população de presas é seguido (com retardo) por um crescimento na população de predadores;

3. Um decréscimo na população de presas é seguido (com retardo) por um decréscimo na população de predadores;

4. Se o número de predadores é pequeno, então o número de presas cresce;

5. Se o número de predadores é grande, então o número de presas diminui;

6. Se o número de presas é grande, então o número de predadores aumenta;

7. Se o número de presas é pequeno, então o número de predadores diminui.

Nesse artigo elaboramos um modelo presa-predador acoplado ao parasitismo para representar a interação entre pulgões (presas/hospedeiros), joaninhas (predadores) e um afidídeo (parasitóide) na citricultura, usando sistemas baseados em regras fuzzy, cujas trajetórias contemplam as sete características comentadas acima.

A seguir, apresentamos um breve resumo da Morte Súbita dos Citros e da interação entre pulgões e joaninhas e pulgões e afidídeos. 
Morte Súbita dos Citros. A Morte Súbita dos Citros é uma doença que tem causado sérios prejuízos aos citricultores, chegando ao ponto de destruir grandes plantações no norte do estado de São Paulo e no sul de Minas Gerais. É uma doença de combinação copa/porta-enxerto e que pode levar à morte plantas sobre porta-enxertos intolerantes. Pesquisadores têm mostrado que os ductos, que levam nutrientes gerados na fotossíntese para as raízes, ficam obstruídos e degenerados. Sem comida, as raízes apodrecem, a árvore decai e morre [2].

Pesquisadores acreditam que essa doença é causado por um vírus transmitido por insetos conhecidos como pulgões (vetor). Os inimigos naturais dos pulgões no Brasil são muitos, destacando-se: a joaninha Cycloneda sanguinea (Coleoptera, Coccinelidae) e o afidídeo Lysiphlebus testaceipes (em Hymenoptera, Aphidiidae).

A joaninha é uma predadora feroz de pulgões. A ocorrência constante de larvas e adultos de joaninhas são importantes no controle de pulgões [8].

Freqüentemente podem ser encontrados pulgões mumificados em razão do parasitismo por Lysiphlebus testaceipes. Eles depositam seus ovos nos pulgões e do ovo eclode uma pequena larva que devora o interior do pulgão. Um pulgão morto pelo parasitóide é facilmente reconhecido, porque torna-se descolorido, rígido e inflado. Pulgões parasitados tornam-se mumificados e os afidídeos adultos emergem por orifícios feitos na porção posterior do corpo do hospedeiro [7].

Na seguinte seção, apresentamos o modelo fuzzy, que vai representar a dinâmica dos insetos de interesse nesse trabalho.

\section{Formulação do Modelo Fuzzy}

Como observado acima, a joaninha preda pulgões enquanto larva e adulta. As fêmeas de afidídeos parasitam pulgões quando adultas. Por outro lado, os pulgões são capturados e/ou parasitados por seus inimigos naturais independentemente da sua fase de vida [4]. Essas são características importantes do fenômeno que serão incorporadas ao modelo. Fatos como esses não são considerados em modelo de predação simples, por exemplo, Lotka-Volterra, onde qualquer tipo de heterogeneidade das populações não é considerado. De acordo com [9], modelos simples não são adequados para estudar relações presa-predador, quando as populações envolvidas podem apresentar diferentes dinâmicas de acordo com sua idade. Cada larva desses predadores pode consumir até 200 pulgões por dia, e o adulto preda, em média, 20 pulgões por dia [6]. Então a população de predadores consistirá de larvas e adultos. Assim, vamos distinguir essas subpopulações e suas particularidades no modelo presa-predador [18].

A partir das informações acima, podemos considerar que os predadores são diferenciados de acordo com seu potencial de predação. O potencial de predação da população de predadores é definido como sendo $P_{y}=p_{1}+0,1 * p_{2}$, onde $p_{1}$ é o número de larvas e $p_{2}$ é o número de adultos [16].

Como pulgões são capturados e/ou parasitados por seus inimigos naturais independentemente da sua fase de vida, a população de presas não será subdivididas, uma vez que a qualidade de ser presa não depende de seu tempo de vida para ser classificada de acordo com sua habilidade de escapar de seus inimigos. 
Finalmente, como somente fêmeas adultas de afidídeos parasitam pulgões, apenas essa parcela da população de parasitóides será considerada em nosso modelo [19].

O modelo fuzzy será composto por um sistema presa-predador e outro sistema acoplado, hospedeiro-parasitóide, onde as bases de regras fuzzy são qualitativamente análogas. As variáveis do sistema presa-predador são número de presas, potencialidade de predadores (entradas) e suas variações (saídas). As variáveis do sistema hospedeiro-parasitóide são número de presas, número de parasitóides (entradas) e suas variações (saídas). Entretanto, um conhecimento preciso a respeito da relação entre as variáveis de estado e suas variações não são disponíveis. Por outro lado, informações qualitativas de especialistas, em particular por entomologistas, nos permite propor regras que relacionem as variáveis de estado com suas variações. De acordo com esses especialistas foram propostas 25 regras do tipo: "SE a quantidade de presas é alta E potencialidade dos predadores é baixa ENTÃO variação da quantidade de presas é baixa positiva $E$ a variação da potencialidade de predadores é positiva"; "SE a quantidade de hospedeiros é baixa E quantidade de parasitóides é alta ENTÃO variação da quantidade de hospedeiros é negativa $E$ a variação da quantidade de parasitóides é alta negativa".

\begin{tabular}{|c|c|c|c|c|c|c|}
\hline & \multicolumn{6}{|c|}{$\mathrm{y}$} \\
\hline \multirow{11}{*}{$\mathrm{x}$} & \multirow{3}{*}{ baixa } & baixa & média baixa & média alta & alta & muito alta \\
\hline & & $\mathrm{x}^{\prime} \mathrm{AP}$ & x'MP & $\mathrm{x}^{\prime} \mathrm{MN}$ & x'MN & x'AN \\
\hline & & $y^{\prime} B P$ & $\mathrm{y}^{\prime} \mathrm{BN}$ & $\mathrm{y}^{\prime} \mathrm{MN}$ & $y^{\prime} \mathrm{AN}$ & $y^{\prime} A N$ \\
\hline & \multirow[t]{2}{*}{ média baixa } & $\mathrm{x}^{\prime} \mathrm{MP}$ & $\mathrm{x}^{\prime} \mathrm{MP}$ & $\mathrm{x}^{\prime} \mathrm{MN}$ & $\mathrm{x}^{\prime} \mathrm{MN}$ & $\mathrm{x}^{\prime} \mathrm{AN}$ \\
\hline & & $\mathrm{y}^{\prime} \mathrm{BP}$ & $\mathrm{y}^{\prime} \mathrm{BN}$ & $\mathrm{y}^{\prime} \mathrm{MN}$ & $\mathrm{y}^{\prime} \mathrm{MN}$ & $\mathrm{y}^{\prime} \mathrm{MN}$ \\
\hline & \multirow[t]{2}{*}{ média alta } & $\mathrm{x}^{\prime} \mathrm{MP}$ & $\mathrm{x}^{\prime} \mathrm{BP}$ & $\mathrm{x}^{\prime} \mathrm{BN}$ & $\mathrm{x}^{\prime} \mathrm{MN}$ & $\mathrm{x}^{\prime} \mathrm{AN}$ \\
\hline & & $y^{\prime}$ MP & $\mathrm{y}^{\prime} \mathrm{BN}$ & $\mathrm{y}^{\prime} \mathrm{BN}$ & $\mathrm{y}^{\prime} \mathrm{MN}$ & $\mathrm{y}^{\prime} \mathrm{MN}$ \\
\hline & \multirow[t]{2}{*}{ alta } & $\mathrm{x}^{\prime} \mathrm{BP}$ & $\mathrm{x}^{\prime} \mathrm{BP}$ & $\mathrm{x}^{\prime} \mathrm{BN}$ & $\mathrm{x}^{\prime} \mathrm{MN}$ & $\mathrm{x}^{\prime} \mathrm{AN}$ \\
\hline & & $\mathrm{y}^{\prime} \mathrm{AP}$ & $y^{\prime} B P$ & $\mathrm{y}^{\prime} \mathrm{BP}$ & $\mathrm{y}^{\prime} \mathrm{BN}$ & $\mathrm{y}^{\prime} \mathrm{BN}$ \\
\hline & \multirow[t]{2}{*}{ muito alta } & $\mathrm{x}^{\prime} \mathrm{BP}$ & $\mathrm{x}^{\prime} \mathrm{BN}$ & $\mathrm{x}^{\prime} \mathrm{MN}$ & $\mathrm{x}^{\prime} \mathrm{MN}$ & $\mathrm{x}^{\prime} \mathrm{AN}$ \\
\hline & & $y^{\prime} \mathrm{AP}$ & $\mathrm{y}^{\prime} \mathrm{MP}$ & y' MP & $y^{\prime} B P$ & $\mathrm{y}^{\prime} \mathrm{BN}$ \\
\hline
\end{tabular}

Tabela 1: Base de regras. $\mathrm{x}=$ quantidade de presas/hospedeiros, $\mathrm{y}=$ potencialidade de predadores/quantidade de parasitóides, x'=variação da quantidade de presas/hospedeiros, $\mathrm{y}^{\prime}=$ variação da potencialidade de predadores/quantidade de parasitóides, $\mathrm{BP}=$ baixa positiva, $\mathrm{MP}=$ média positiva, $\mathrm{AP}=$ alta positiva, $\mathrm{BN}=$ baixa negativa, $\mathrm{MN}=$ média negativa, $\mathrm{AN}=$ alta negativa.

Essa base de regras é coerente com os modelos clássicos determinísticos do tipo presa-predador ([22], [21]). E como veremos, as soluções são compatíveis com esses mesmos modelos.

A partir do Método de Inferência de Mamdani e a defuzzificação do Centro de Gravidade, obtemos as taxas de variações das populações. Em cada instante $t$, o 
número de presas e o potencial de predação são dados pelas fórmulas

$$
\left\{\begin{array}{l}
x(t)=x\left(t_{0}\right)+\int_{t_{0}}^{t} x^{\prime}(s) d s \\
P_{y}(t)=P_{y}\left(t_{0}\right)+\int_{t_{0}}^{t} P_{y}^{\prime}(s) d s
\end{array}\right.
$$

e o número de hospedeiros e de parasitóides são dados pelas fórmulas

$$
\left\{\begin{array}{l}
x(t)=x\left(t_{0}\right)+\int_{t_{0}}^{t} x^{\prime}(s) d s \\
z(t)=z\left(t_{0}\right)+\int_{t_{0}}^{t} z^{\prime}(s) d s
\end{array}\right.
$$

Nas simulações numéricas procuramos observar a variação do número de presas/hospedeiros, potencial de predação e número de parasitóides. Para isso, consideramos um número inicial de pulgões, $x_{0}$, um número inicial de potencial de predação, $P_{y_{0}}$, e um número inicial de parasitóides, $z_{0}$, num ramo de uma árvore. A partir das condições iniciais, o sistema fuzzy gera $x^{\prime}$ e $P_{y}^{\prime}$ como saídas. Desses dois últimos valores, para obter $x$ e $P_{y}$ em cada iteração fazemos

$$
\left\{\begin{array}{l}
x\left(t_{i+1}\right)=x\left(t_{i}\right)+h x^{\prime}(s) \\
P_{y}\left(t_{i+1}\right)=P_{y}\left(t_{i}\right)+h P_{y}^{\prime}(s)
\end{array}\right.
$$

onde $h$ é o incremento, e finalmente,

$$
\left\{\begin{array}{l}
x\left(t_{i+1}\right)=x\left(t_{i}\right)+h x^{\prime}(s) \\
z\left(t_{i+1}\right)=z\left(t_{i}\right)+h z^{\prime}(s)
\end{array}\right.
$$

Usando (4.3) e (4.4), e iniciando com $t_{i}=t_{0}+i$ e $t_{0}=0$, obtemos os valores de $x, P_{y}$ e $z$, e assim, sucessivamente.

Simulações de trajetórias produzidas pelo modelo fuzzy segue dos passos abaixo:

- É dada uma população inicial de presas $\left(x\left(t_{0}\right)\right)$ e um número inicial para o potencial de predação $\left(P_{y}\left(t_{0}\right)\right)$ como dados de entrada do sistema baseado em regras fuzzy do tipo presa-predador;

- O sistema baseado em regras fuzzy do tipo presa-predador fornece os valores de saída: $x^{\prime}\left(t_{1}\right)$ e $P_{y}\left(t_{1}\right)^{\prime}$

- De (4.3), encontramos $x\left(t_{1}\right)$ e $P_{y}\left(t_{1}\right)$;

- Tendo um número de presas $\left(x\left(t_{1}\right)\right)$ e dado um número inicial de parasitóides $\left(z\left(t_{0}\right)\right)$ como dados de entrada do sistema baseado em regras fuzzy do tipo hospedeiro-parasitóide;

- O sistema baseado em regras fuzzy do tipo hospedeiro-parasitóide fornece os valores de saída: $x^{\prime}\left(t_{2}\right)$ e $z^{\prime}\left(t_{1}\right)$;

- De (4.4), encontramos $x\left(t_{2}\right)$ e $z\left(t_{1}\right)$; 
- $x\left(t_{2}\right), P_{y}\left(t_{1}\right)$ e $z\left(t_{1}\right)$ são as variáveis de entrada do sistema baseado em regras fuzzy tipo presa-predador e assim sucessivamente.

A evolução dos contingentes populacionais de pulgões, tanto na ausência como na presença de seus inimigos estão ilustados nas Figuras 2 e 3.

Pode-se notar claramente que a presença de predadores e parasitóides é decisiva para a contenção da população de pulgões.

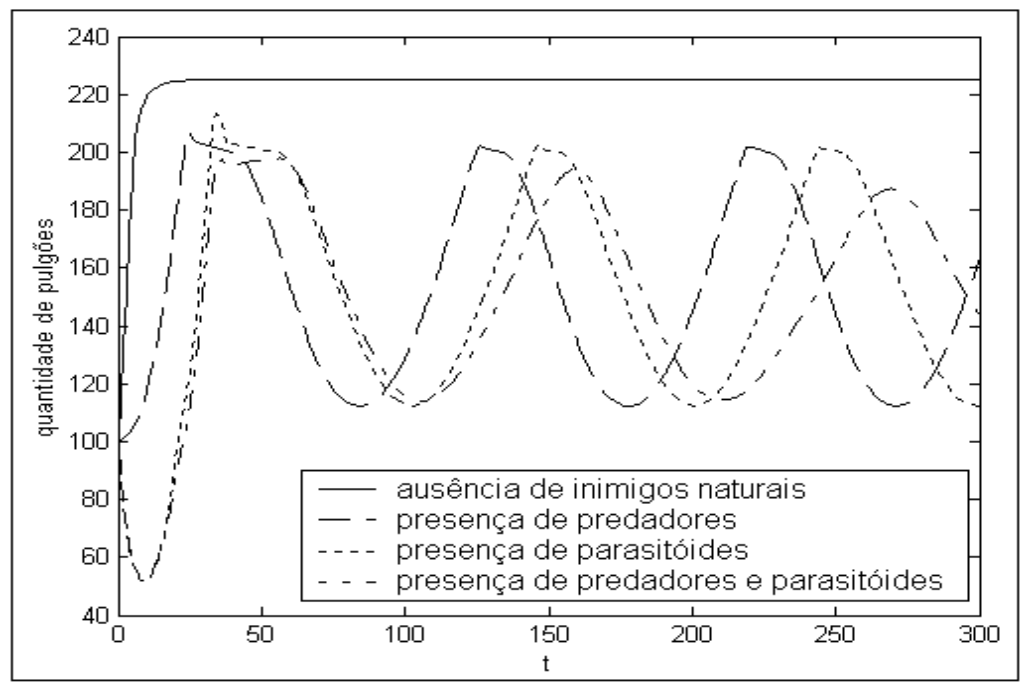

Figura 2: Evolução no tempo dos contingentes populacionais de pulgões na ausência de seus inimigos $\left(x_{0}=100, P_{y 0}=0, z_{0}=0\right)$, de pulgões na presença de predadores $\left(x_{0}=100\right.$, $\left.P_{y 0}=3,2, z_{0}=0\right)$, de pulgões na presença de parasitóides $\left(x_{0}=100, P_{y 0}=0, z_{0}=10\right)$ e de pulgões na presença de ambos $\left(x_{0}=100, P_{y 0}=3,2, z_{0}=10\right)$ utilizando o modelo fuzzy.

\section{Conclusões}

Esse artigo sugere que a utilização da Lógica Fuzzy em Ecologia pode ser uma ferramenta capaz de representar a interação entre espécies em um ambiente onde as informações disponíveis são apenas qualitativas. Através de sistemas baseados em regras fuzzy foi possível modelar a dinâmica pulgões-joaninhas-parasitóides sem o uso de equações diferenciais, uma vez que o campo de direções deve ser rigorosamente conhecido. Por outro lado, se optássemos por modelar nosso problema utilizando sistemas determinísticos, como aqueles dados por equações diferenciais ou diferenças, seria necessário conhecermos todos os parâmetros dessas equações, o que não temos disponível. Por isso, consideramos a Teoria dos Conjuntos Fuzzy como uma grande contribuição na construção de modelos matemáticos, principalmente quando alguns parâmetros das equações diferenciais não são conhecidos. Entretanto, se houver necessidade, esses parâmetros podem ser obtidos por meio de 


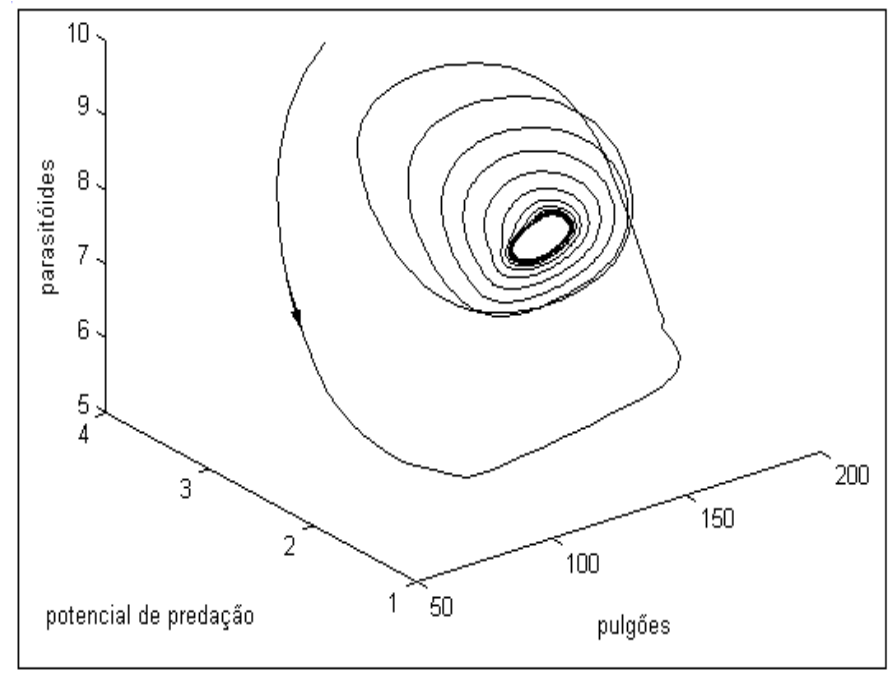

Figura 3: Espaço de fase do modelo fuzzy, com $x_{0}=100, P_{y 0}=3,2$ e $z=10$.

ajuste da curva solução dada pelo modelo baseado em regras fuzzy [17]. E, mesmo sem as tradicionais equações diferenciais, podemos analisar a existência de pontos de equilíbrio e estabilidade de sistemas p-fuzzy como este [20].

Embora já comentado acima, gostaríamos de enfatizar as vantagens do uso de modelos baseados em regras fuzzy em ecologia:

- o modelo fuzzy não requer a utilização de parâmetros em sua formulação;

- no sistema fuzzy, usa-se uma base de regras, ao invés de equações, eliminando a exigência de um campo de direções precisamente fornecido;

- diferentemente de modelos clássicos dados por equações diferenciais, os conjunto de entrada e de saída e a base de regras fuzzy podem ser construídos por especialistas da área, indicando a participação direta do especialista no modelo matemático;

- a facilidade do método matemático na sua formulação e, consequentemente, uma análise de sua eficiência por parte do especialista (ecólogo) do fenômeno;

- pode-se obter critérios de estabilidade para pontos de equilíbrio, compatíveis com dos modelos determinísticos correspondentes [20].

\section{Agradecimentos}

Os autores agradecem ao Dr. Renato Beozzo Bassanezi (Fundecitrus-Araraquara/SP-Brasil), Dr. Arício Xavier Linhares (IB/UNICAMP-Campinas/SP-Brasil) e Dr. Carlos Roberto Sousa e Silva (DEBE/UFSCar-São Carlos/SP-Brasil).

Abstract. In this work we have used fuzzy rule-based systems to elaborate a predator-prey type of model connected to parasitism to study the interaction among 
aphids (prey/host), ladybugs (predator) and a parasitoid in citriculture, where the aphids are considered as transmitter agents of the Citrus Sudden Death (CSD). Simulations were performed and a graph was drawn to show the prey/host population, the potentiality of the predators and the parasitoid population, and a phase-space.

\section{Referências}

[1] L.C. Barros, R.C. Bassanezi, "Tópicos de Lógica Fuzzy com Aplicações em Biomatemática", vol. 5, IMECC, 2006.

[2] R.B. Bassanezi, A. Bergamin Filho, L. Amorim, N. Gimenes-Fernandes, T.R. Gottwald, Spatial and temporal analysis of Citrus Sudden Death as a tool to generate hypothesis its etiology, Phytopathology, 93 (2003), 502-512.

[3] M. Began, M. Mortimer, "Population Ecology", Blackwell Scientific Publications, 1989.

[4] A. Braga, C.R. Sousa-Silva, "Afídeos de citros (Citrus sinensis) e seus predadores na região de São Carlos-SP", Monografia, Departamento de Ecologia e Biologia Evolutiva, Universidade Federal de São Carlos, São Carlos, SP, 1999.

[5] L. Edelstein-Keshet, "Mathematical Models in Biology", McGraw-Hill, Inc., 1987.

[6] S. Gravena, O controle biológico na cultura algodoeira. Informe Agropecuário, 9 (2003), 3-15.

[7] K.S. Hagen, J.A. McMurty, "Biological control and insect pest management (Natural enemies and predator-prey ratios)", 28-40, University of California, 1979 .

[8] I. Hodek, "Biology of Coccinellidae", Prague, Academic of Science, 1973.

[9] C. Hsin, T. Yang, Two-sex life table and predation rate of Propylaea japonica Thunberg (Coleoptera: Coccinellidae) Fed on Myzus persicae (Sulzer) (Homoptera: Aphididae), Environmental Entomology, 32, No. 2 (2003), 327-333.

[10] R.M. Jafelice, L.C. Barros, R.C. Bassanezi, F. Gomide, Fuzzy modeling in symptomatic HIV virus infected population, Bulletin of Mathematical Biology, 66 (2003), 1597-1620.

[11] G. Klir, B. Yuan, "Fuzzy Sets and Fuzzy Logic - Theory and Application", Prentice Hall, 1995.

[12] J. Morales, V. Buranr Jr., Interactions between Cycloneda sanhuine and the Brown Citrus Aphid: Adult Feeding and Larval Mortality, Environmental Entomology, 14, No. 4 (1985), 520-522.

[13] J. Murray, "Mathematical Biology", Berlin, Springer, 1990. 
[14] J.R.P. Parra, H.N. Oliveira, A.S. Pinto, "Guia Ilustrado de Pragas e Insetos benéficos dos citros", Esalq-USP, Piracicaba, SP, 2003.

[15] W. Pedrycz, F. Gomide, "An Introduction to Fuzzy Sets: Analysis and Design", Massachusetts Institute of Technology, 1998.

[16] M.S. Peixoto, "Sistemas Dinâmicos e Controladores Fuzzy: um Estudo da Dispersão da Morte Súbita dos Citros em São Paulo", Tese de Doutorado, IMECC-UNICAMP, Campinas, SP, 2005.

[17] M.S. Peixoto, L.C. Barros, R.C. Bassanezi, Predator-prey fuzzy model, Bulletin of Mathematical Biology, (aceito).

[18] M.S. Peixoto, L.C. Barros, R.C. Bassanezi, Predator-prey fuzzy model, in "Citrus: Aphids and Ladybugs", Proceedings of the Fourth Brazilian Symposium on Mathematical and Computational Biology / First International Symposium on Mathematical and Computational Biology, I (2005), 228-239.

[19] S.M.M. Rodrigues, V.H.P. Bueno, Parasitism rates of Lysiphlebus testaceipes(Cresson) (Hym.:Aphidiidae) on Schizaphis graminium (Rond.) and Aphis gossypii Glover (Hem.:Aphididae), Neotropical Entomology, 30, No. 4, 2001.

[20] J.D.M. Silva, "Análise de Estabilidade de Sistemas Dinâmicos P-fuzzy com Aplicações em Biomatemática", Tese de Doutorado, IMECC-UNICAMP, Campinas, SP, 2005.

[21] Y.M. Svirezhev, D.O. Logofet, "Stability of Biological Communities", Mir Publishers, Moscow, 1983.

[22] J.T. Tanner, The Stability and the Intrinsic Growth Rates of Prey and Predator Populations, Ecology, 56 (1975), 855-867.

[23] L.A. Zadeh, Fuzzy Sets, Informat. Control, 8 (1965), 338-353. 